\title{
Magnetic Resonance Evaluation of Pelvic Mass Lesions in Females with Histopathological Correlation: An Institutional Experience
}

\author{
Reddy Ravikanth ${ }^{1}$ \\ ${ }^{1}$ Department of Radiology, Holy Family Hospital, Thodupuzha, \\ Kerala, India
}

Asian J Oncol 2019;5:44-49

\begin{abstract}
Address for correspondence Reddy Ravikanth, MD, Department of Radiology, Holy Family Hospital, Thodupuzha 685605, Kerala, India (e-mail: ravikanthreddy06@gmail.com).
\end{abstract}

\begin{abstract}
Keywords

- MRI

- pelvic mass lesions

- malignancy

Introduction Magnetic resonance imaging (MRI) is often used in the detection and staging of large pelvic masses. Many large masses in the female pelvis arise from the reproductive organs. These pelvic masses most commonly arise from the uterus, cervix, ovaries, and fallopian tubes.

Objective This study was aimed to assess the role of MRI in female pelvic mass lesions. Also, it presents a pictorial review of MRI images of such pelvic masses.

Materials and Methods This study was conducted on 50 female patients with clinically suspected pelvic masses at physical examination and referred for MRI at a tertiary care hospital over a 2-year period between July 2017 and June 2019.

Results Most common lesions evaluated on imaging were benign uterine lesions (15 cases, 34.09\%), benign adnexal lesions (13 cases, 29.54\%), malignant adnexal (10 cases, $22.73 \%$ ), malignant uterine cervical lesions (6 cases, $13.64 \%$ ); two cases had indeterminate type lesions, two lesions proved to be normal bowel loops on MRI, and in two cases MRI could not be performed. Cystic lesions were commonly seen in adnexa (15 out 21 ) while solid lesions were common in uterine cervical region (18 out 23). Out of these, 14 were complex cystic adnexal masses and 10 were malignant. Uterine cervical lesions were carcinoma cervix. Better assessment with improved imaging capability was possible on MRI for invasion of surrounding structures in 10 cases, lymphadenopathy in 3 cases, ascites in 13 cases, peritoneal implant in 6 cases, encasement in 1 case, and distant metastases in 1 case.

Conclusion In conclusion, pelvic mass lesions in females are more common above the age of 45 years. Adnexal masses are usually cystic, while the uterine masses are solid in texture. As the complexity and size of the cystic adnexal masses increases, there are increased chances of malignancy. Pretreatment staging and assessment of malignancy, invasion of surrounding structures, encasement, invasion of vessels or assessment of lymphadenopathy, peritoneal implant, ascites, and distant metastases are better appreciated by MRI.
\end{abstract}

\section{Introduction}

Magnetic resonance imaging (MRI) has become an important modality in the evaluation of female pelvic lesions. ${ }^{1}$ Ultrasonography (USG) remains the examination of choice for initial assessment of patients with suspected benign gynecological

received

July 11,2019

accepted

October 23, 2019
DOI https://doi.org/

$10.1055 / \mathrm{s}-0039-3400710$

ISSN 2454-6798. disease, given its relative safety and lower cost. USG may be limited by technical considerations (operator dependence, patient's body habitus, low signal-to-noise ratio, and is inadequate in staging pelvic malignancies). MRI is safe because of no radiation exposure, while CT has a danger of radiation exposure. MRI is considered as the next step in the imaging 
assessment of benign disease of the uterus and adnexa and is becoming the primary imaging modality for evaluating gynecologic malignancies. The multiplaner imaging capability of MRI, superior soft tissue contrast and large field of view offer distinct advantages over both USG and CT (computed tomography) in the assessment of gynecologic abnormalities. Although USG remains the initial modality used in evaluating clinically suspected gynecologic disease. MRI can offer supplemental diagnostic information in cases of suboptimal or equivocal ultrasound examination, and in patients in whom there is discrepancy between sonographic findings and physical examinations. ${ }^{2}$ Endovaginal USG provide improved spatial resolution. USG is not as useful as CT and MRI in the detection of the locoregional extent of pelvic cancer. Generally uterine masses are mainly solid as opposed to ovarian masses which are mainly cystic. ${ }^{3}$ If the mass can be shown to arise from the uterus, it is usually a benign leiomyoma. Leiomyomas are common differential diagnosis of solid adnexal mass. Frequently fibroids can be diagnosed sonographically by their decreased echogenecity and decreased sound through transmission, even though the relationship to the uterus is obscured. Occasionally, it may be impossible to determine the exact origin of the mass by the sonography, instead MRI may be helpful. A tailored MRI may be used to help clarify and confirm cases that are not as definitive sonographically. On MRI, fibroids may also present variable appearances. They are usually low signal such that pedunculated ones can mimic feces. ${ }^{4}$ However, degenerated fibroids can have moderately high signal intensity on T2-weighted (T2-W) scans. There may even be a peculiar nodular appearance to massive leiomyomas that can mimic an ovarian malignancy. The definitive diagnosis can be made by showing the stretching of myometrium around the base of lesion. This, the most definite sign, is much better appreciated on MRI than USG.

\section{Materials and Methods}

This study was conducted on 50 female patients with clinically suspected pelvic masses at physical examination and referred for MRI at a tertiary care hospital over the 2-year period between July 2017 and June 2019. Before patients were recruited, the study protocol was approved by the institutional ethics committee, in accordance with the ethical principles for human investigation outlined by the Second Declaration of Helsinki, and written informed consent was obtained from all patients. MRI examinations were performed using a $1.5 \mathrm{~T}$ scanner (Signa, General Electric Medical Systems, Milwaukee, Wisconsin, United States). Patients were imaged on MRI with an empty bladder after fasting for at least 4 hours. Imaging was performed with the patient supine. In both T1-W and T2-W MRI in axial, sagittal, and coronal planes were evaluated. T1-W images offer excellent contrast between the pelvic organs and adjacent fat allow optimal detection of lymph nodes and are necessary for tissue and fluid characterization (essential for hemorrhagic and fat containing lesions). T2-W image sequences are needed to demonstrate the zonal anatomy of the uterus and vagina and to facilitate the identification of normal ovaries. In addition, T2-W images are usually superior in depicting pathologic conditions of uterus and ovaries. Additional MRI included selective application of fat saturation imaging. It was done to differentiate fat, blood, and proteinaceous fluid in hyper intense lesions in T1-W images. For pelvic MRI axial, sagittal, and coronal fast spin echo T1-W images were obtained by using echo time (TE)-minimal full, repetition time (TR) used was approximately $560 \mathrm{~ms}$, echo train length was 3 , band width was approximately 2.83 in most of the cases. Slice thickness was $5 \mathrm{~mm}$, spacing was approximately $1 \mathrm{~mm}$. Frequency, phase, and number of excitation was adjusted according to the image quality. Frequency direction in axial, sagittal images is anterior/posterior, while in coronal images it is superior/inferior. Patient is kept supine in feet first position. Images were obtained in two-dimensional (2D) mode with a body array coil. In T2-W fast-spin echo images TE and TR are high in this study TE used was 102 and TR was approximately 4,200 , echo train length was 12 , band width was 15.6, slice thickness, spacing, patient's position, mode of imaging, and coil remained the same as that of T1-W images. In fat saturated images, the protocol was same as that of $\mathrm{T} 2-\mathrm{W}$ sequences except for the fat suppression option was selected. Imaging features on MRI forms the mainstay in the differential diagnosis of the pelvic masses. These include shape, size, margins, internal architecture (cystic/solid), organ of origin, extent of involvement of surrounding structures, absence or extent of metastatic involvement, and presence of ascites or peritoneal implant. These features on complete assessment can help to make a diagnosis or at least narrow down the differential diagnosis. Information collected was tabulated in master chart to analyze observations and then statistical analysis of the data on the basis of history, MRI findings were done. Cases were followed-up; histopathological diagnosis was considered final and was obtained by biopsy or fine needle aspiration cytology of mass.

\section{Statistical Analysis}

Descriptive statistics were reported using numbers and percentages for categorical variables. Analysis was done using Microsoft Excel 2013, Microsoft Corp., Redmond, Washington, United States and SPSS Statistical Package Version 20.0, IBM Corp., Armonk, New York, United States.

\section{Results}

Commonest pelvic lesions were solid in consistency (37.5\%) in the age group $>45$ years. ( - Table 1 ) Adnexal mass lesions were commonly benign comprising $66.67 \%$ and malignant in $33.3 \%$ cases (-Table 2). Most common lesions evaluated on imaging were benign uterine lesions (15 cases, 34.09\%), benign adnexal lesions(13 cases, 29.54\%), malignant adnexal (10 cases, 22.73\%), malignant uterine cervical lesions (6 cases, 13.64\%); two cases had indeterminate type lesions, two lesions proved to be normal bowel loops on MRI, and in two cases MRI could not be performed. Cystic lesions were commonly seen in adnexa (15 of 21), while solid lesions were common in uterine cervical region (18 of 23). Among these, 14 were complex cystic adnexal masses and 10 were 
Table 1 Comparison of pelvic masses according to internal consistency on MRI

\begin{tabular}{|l|l|l|l|l|}
\hline \multirow{2}{*}{ Age $(\mathbf{y})$} & \multicolumn{5}{|c|}{ MRI $(\boldsymbol{n}=48[\%])$} \\
\cline { 2 - 5 } & Cystic & Solid & Complex & Others \\
\hline$<35$ & $3(6.25)$ & $4(8.33)$ & $3(6.25)$ & $1(2.08)$ \\
\hline $35-44$ & $4(8.33)$ & $6(12.50)$ & $4(8.33)$ & $0(0.00)$ \\
\hline$>45$ & $6(12.50)$ & $8(16.67)$ & $8(16.67)$ & $8(16.67)$ \\
\hline Total & $13(27.08)$ & $18(37.50)$ & $15(31.25)$ & $8(16.67)$ \\
\hline
\end{tabular}

Abbreviation: MRI, magnetic resonance imaging.

Table 2 Distribution of adnexal masses according to size on MRI

\begin{tabular}{|l|l|l|l|}
\hline \multirow{2}{*}{ Pelvic mass $(\mathrm{cm})$} & \multicolumn{3}{|c|}{ MRI $(\boldsymbol{n}=48[\%])$} \\
\cline { 2 - 4 } & Benign & Malignant & Total \\
\hline$<5$ & $11(22.92)$ & $3(6.25)$ & $14(29.17)$ \\
\hline$>5$ & $21(43.75)$ & $13(27.08)$ & $34(70.83)$ \\
\hline Total & $32(66.67)$ & $16(33.33)$ & $48(100.00)$ \\
\hline
\end{tabular}

Abbreviation: MRI, magnetic resonance imaging.

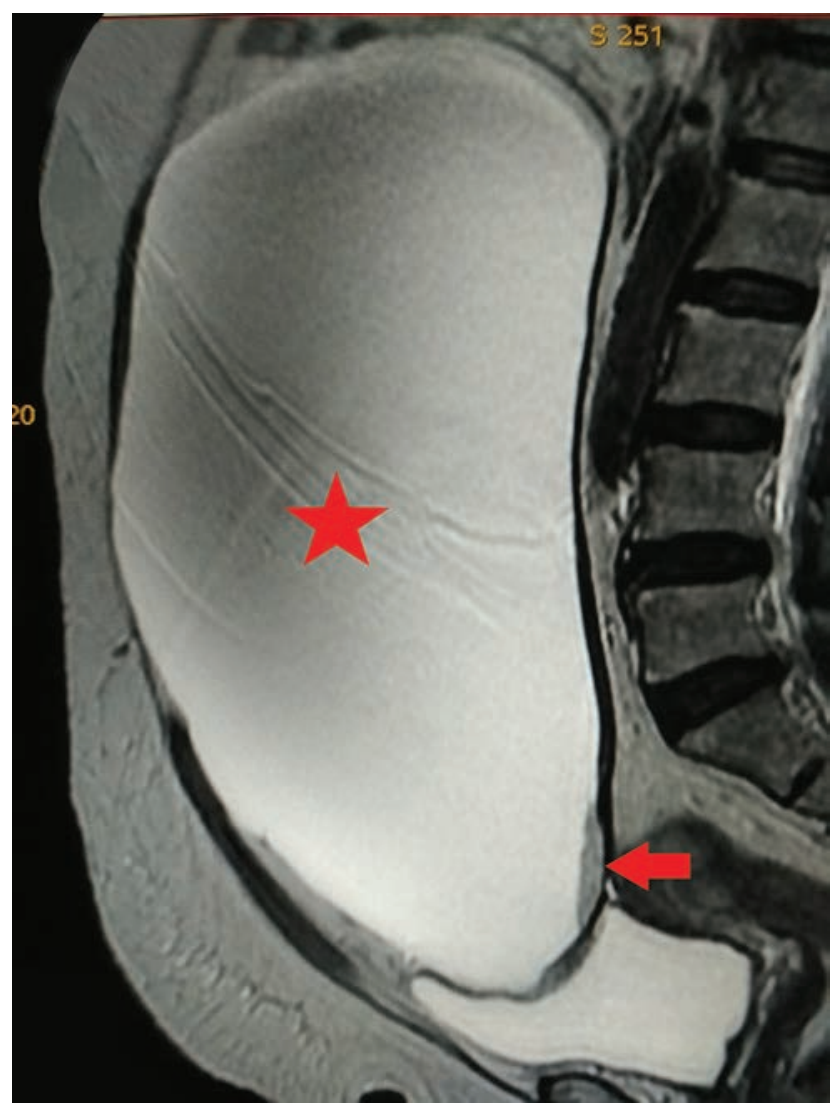

Fig. 1 Sagittal T2-W MRI demonstrating a large well-defined thin walled unilocular cystic lesion (star) arising from right ovary. Small mural nodule is visualized (arrow). Findings could represent right ovarian cystic neoplasm (possible ovarian serous cystadenoma). MRI, magnetic resonance imaging; T2-W, T2-weighted.

malignant. Uterine cervical lesions were carcinoma cervix. In cases of larger lesions, MRI was the best. Two cases of dermoid were diagnosed confidently on the MRI because of superior soft tissue contrast and fat detection. By MRI, better assessment with improved imaging capability was possible

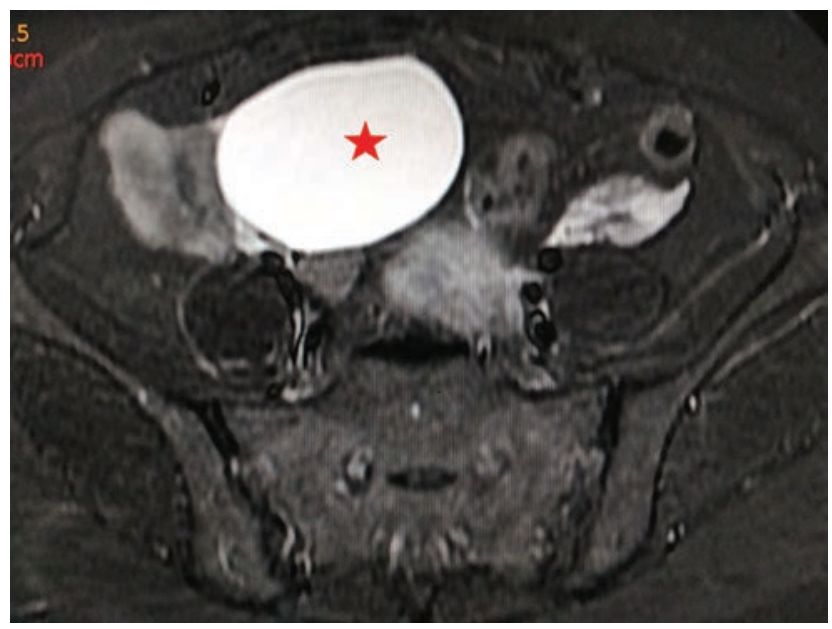

Fig. 2 Axial STIR MRI demonstrating a well-defined nonenhancing thin-walled cystic lesion (star) arising from the right adnexa, features are of concern for ovarian etiology, likely serous cystadenoma. MRI, magnetic resonance imaging.

in invasion of surrounding structure in 10 cases, lymphadenopathy in 3 cases, ascites in 13 cases, peritoneal implant in 6 cases, encasement in 1 case, and distant metastases in 1 case. Ascites and metastases were diagnosed in all the patients on USG. Results indicated higher diagnostic capability of MRI for dermoid, hemorrhagic cyst, and pretreatment assessment of pelvic malignancies (-Figs. 1-13).

\section{Discussion}

Evaluation of site of origin and morphology was possible in $42 \%$ cases, while on MRI it was possible in $93.75 \%$ cases. All malignant adnexal masses were more than $5 \mathrm{~cm}$ in size, 10 out of 13 benign adnexal masses were more than $5 \mathrm{~cm}$ in size which is contrary to the study done by Meire et al. ${ }^{5}$ In his study he found that cysts $<5 \mathrm{~cm}$ with thin septa and no solid nodules were generally benign. Regarding the accuracy of MRI for the 


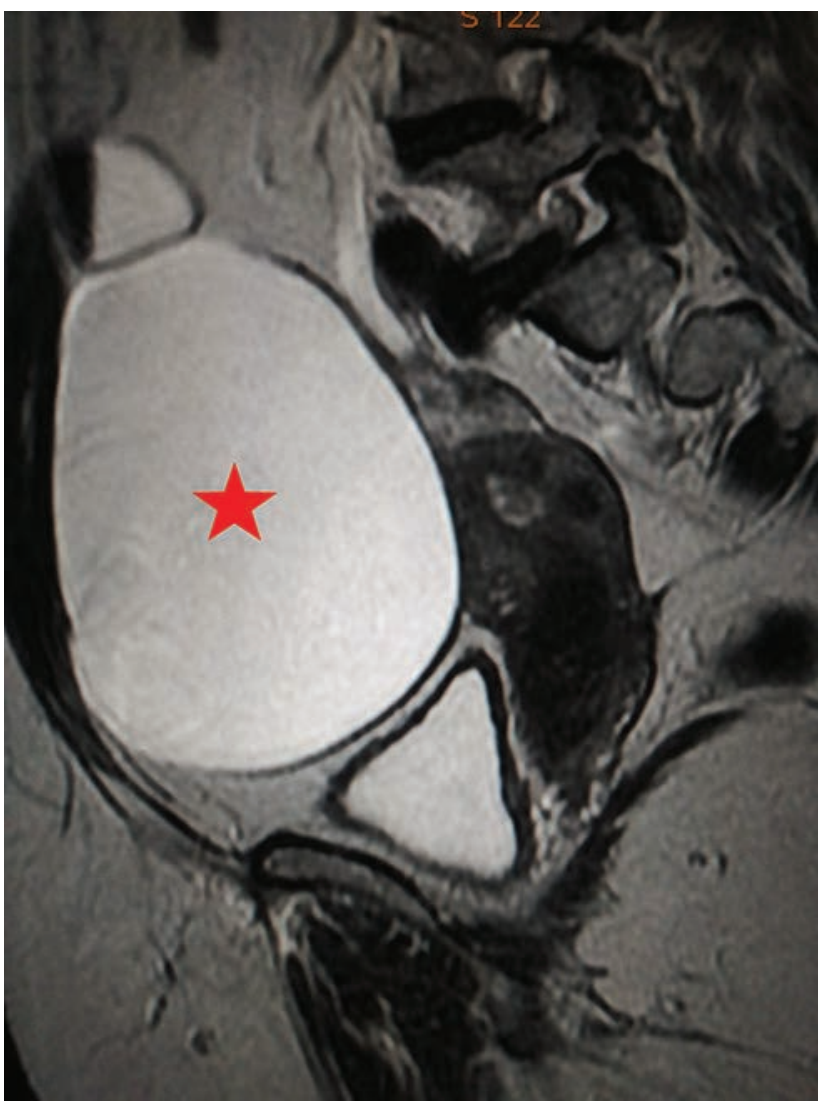

Fig. 3 Sagittal T2-W MRI demonstrating a large well-defined thinwalled cystic abdomino pelvic lesion (star) with no solid component. MRI, magnetic resonance imaging.

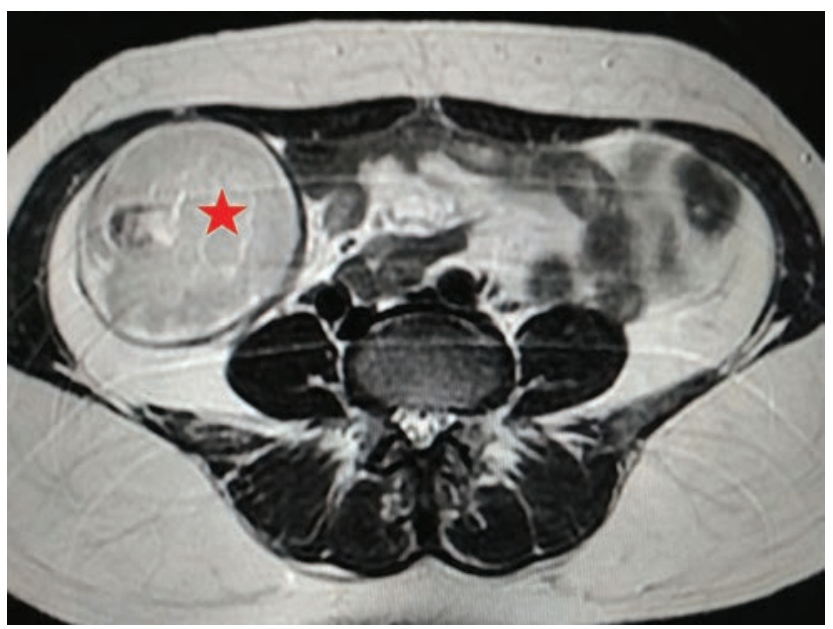

Fig. 4 Axial T2-W MRI demonstrating a well-defined mixed signal intensity lesion (star) at right adnexa with few areas of fat fluid levels, foci low signal calcification and nodule in the wall of the right mass, $\mathrm{S} / \mathrm{O}$ benign cystic teratoma. MRI, magnetic resonance imaging.

diagnosis of pelvic masses, on MRI correct evaluation of site of origin and morphology was possible in $93.75 \%$ cases. MRI provided new information in $48 \%$ cases. This information is related to the origin of mass, spread of tumor, and internal consistency (such as fat or hemorrhagic contents within the lesion) in most

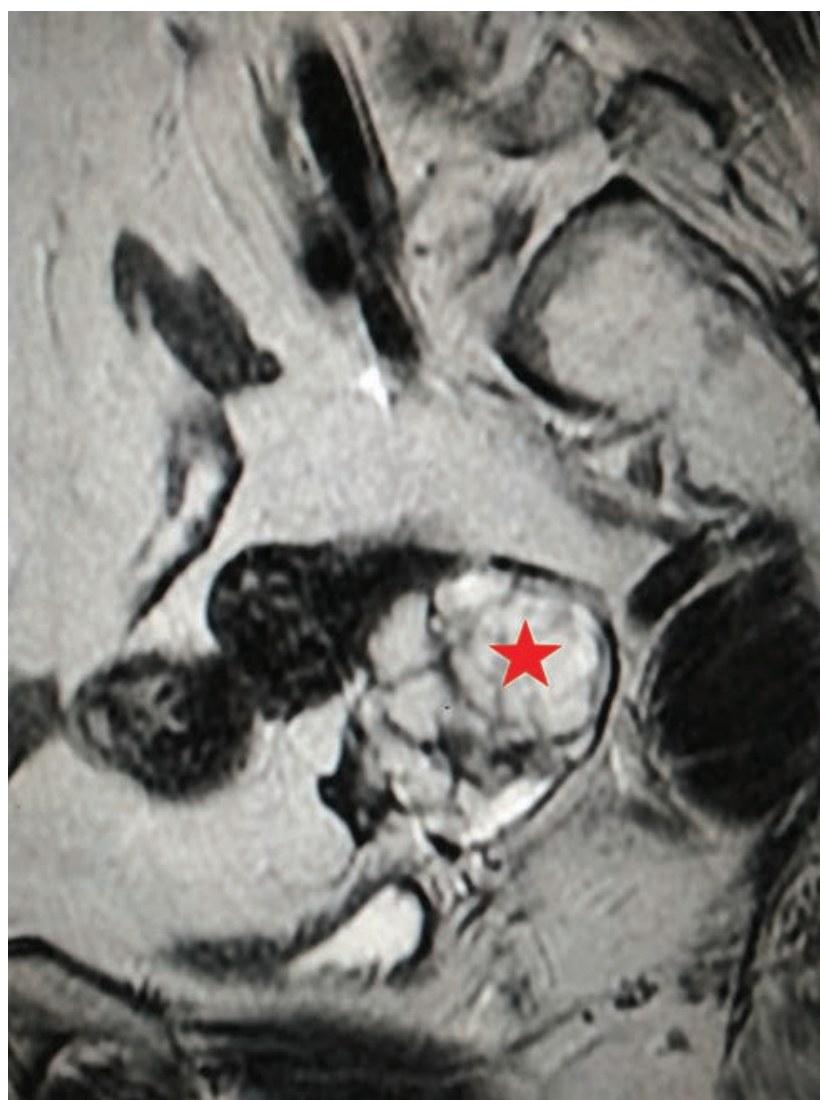

Fig. 5 Sagittal T2-W MRI demonstrating a well-defined multiloculated heterogeneously hyperintense cystic lesion (star) in the right adnexa. Right ovary is not visualized separately from the lesion, features are of concern for malignant ovarian etiology. MRI, magnetic resonance imaging.

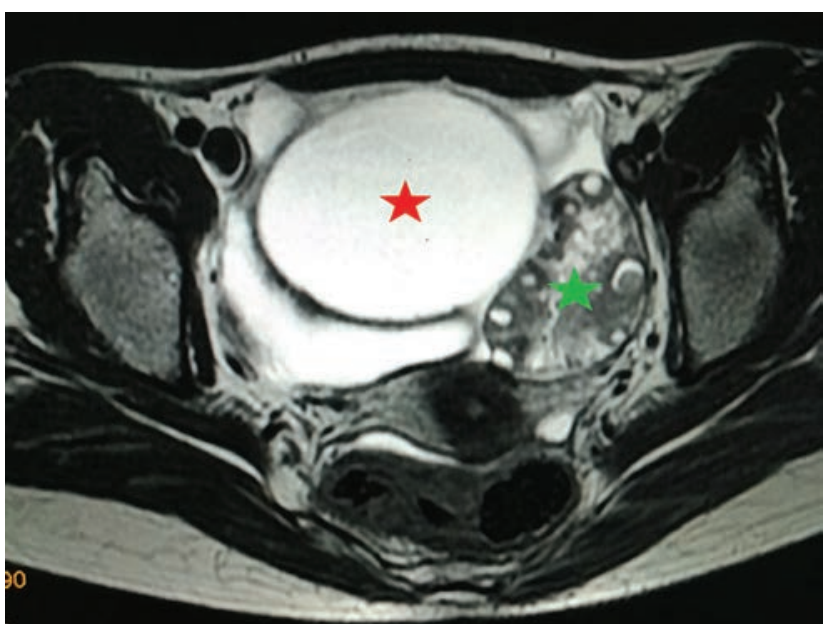

Fig. 6 Axial T2-W MRI demonstrating a well-defined hyperintense left paraovarian cystic lesion (red star) with twisted ovarian pedicle and bulky left ovary (green star), possibly torsion of left ovary. MRI, magnetic resonance imaging.

of the cases. By MRI, presence of fat was detected in $4 \%$ cases. Heterogeneous lesions without fat contents were seen in $24 \%$ cases and were due to tiny hemorrhagic foci. These findings correlate very well with Jain and Jeffery ${ }^{6}$ who concluded that MRI is superior in detection and differentiation of hemorrhagic 


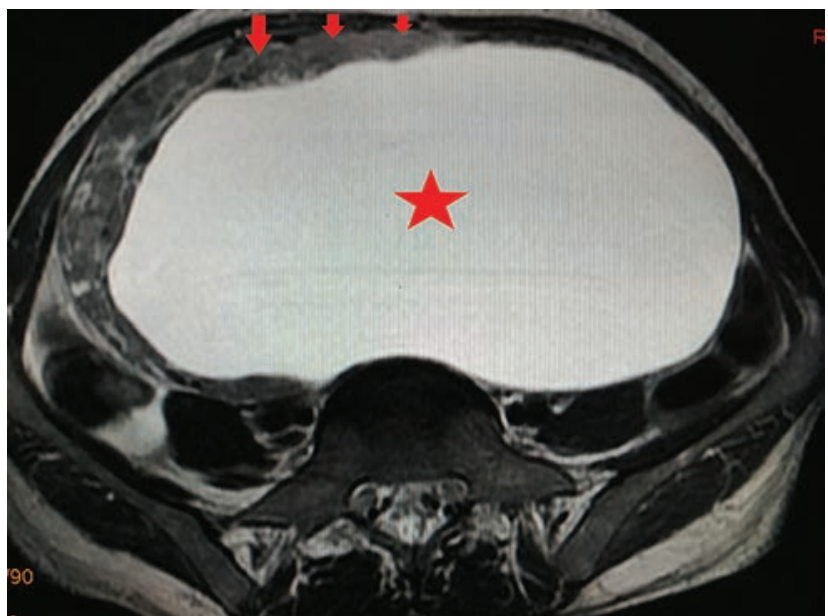

Fig. 7 Axial T2-W MRI demonstrating a well-defined hyperintense adnexal cyst (star) of ovarian origin. Mural nodule (arrows) is seen anterolaterally. MRI, magnetic resonance imaging.

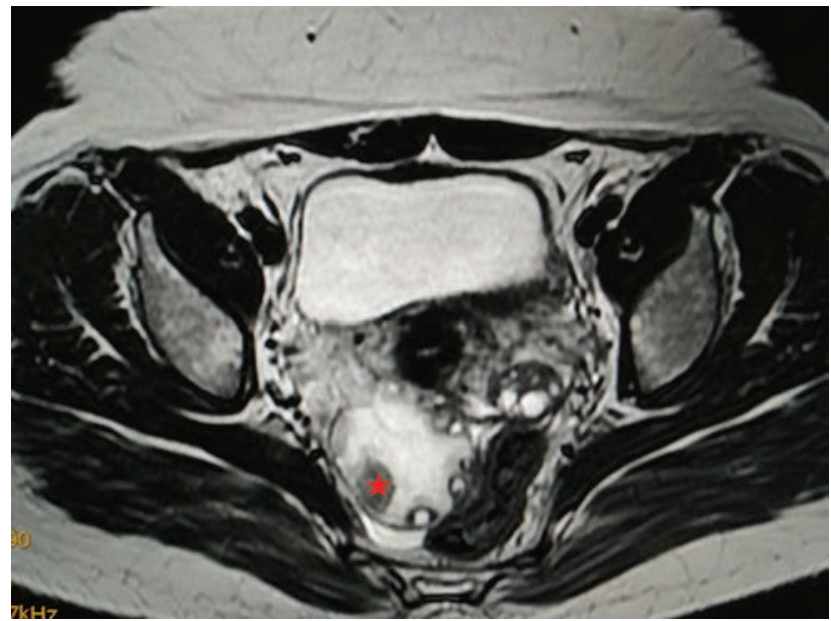

Fig. 8 Axial T2-W MRI demonstrating bulky right ovary with well-defined hypointense solid lesion (star) and twisted ovarian pedicle features are of concern for neoplastic lesion of right ovary with probable torsion. MRI, magnetic resonance imaging.

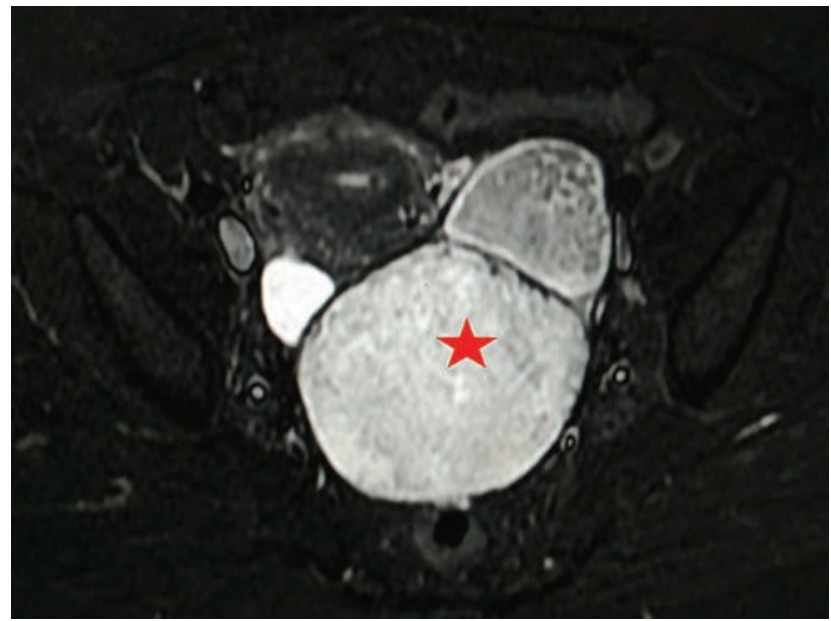

Fig. 9 Axial STIR MRI demonstrating a thin walled heterogeneously hyperintense lesion (star) in the left adnexa: findings are of concern for atypical teratoma versus malignant etiology. MRI, magnetic resonance imaging.

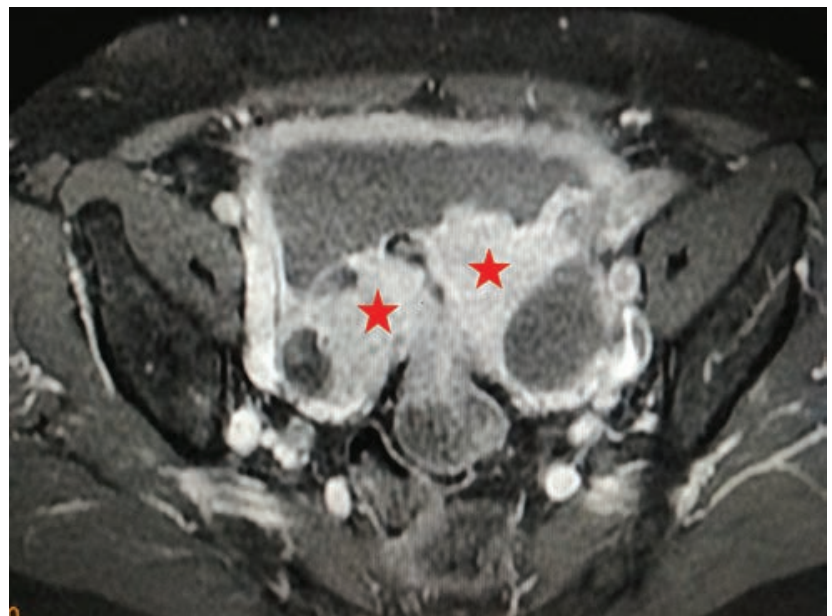

Fig. 10 Axial T1 + contrast MRI demonstrating bilateral lobulated solid cystic lesions (stars) in bilateral adnexal regions. The solid component shows heterogeneous enhancement on post contrast images. Bilateral ovaries are not visualized separately, features are of concern for malignant ovarian etiology. MRI, magnetic resonance imaging.

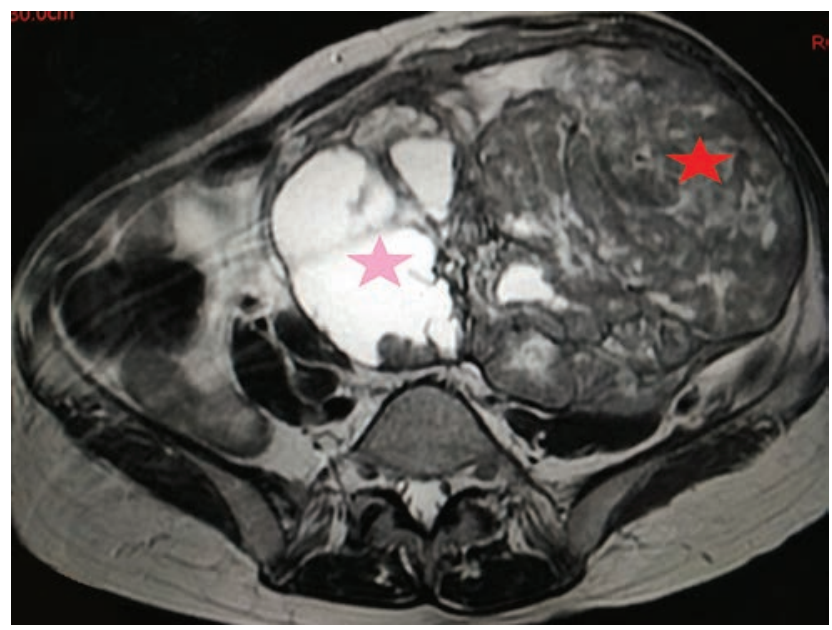

Fig. 11 Axial T2-W MRI demonstrating a large complex solid (red star) cystic (pink star) mass lesion involving pelvis and left lower abdomen. Both ovaries not separately seen, features are consistent with ovarian neoplastic etiology. MRI, magnetic resonance imaging.

masses from the dermoids (fat containing masses) when fat suppression technique was used. Troiano et $\mathrm{al}^{7}$ found that MRI is $100 \%$ sensitive and $99 \%$ specific in prospective diagnosis of dermoid.

MRI demonstrated the internal architecture of hemorrhagic cyst as multiple fine septations, thereby allowing a specific diagnosis. Although MRI allowed identification of blood on the basis of characteristic signal intensity on T1- and T2-W images and contributed to a diagnosis of hemorrhagic mass, identification of a focal hemorrhagic mass does not allow a specific diagnosis of hemorrhagic cyst. MRI is accurate in the staging of malignant lesions which involve the determination of encasement of vessels. It was detected in $2 \%$ cases; invasion was detected in $20 \%$ cases, pelvic lymphadenopathy in $6 \%$, peritoneal implants in $12 \%$, ascites in $26 \%$, and distant metastasis in $2 \%$ cases. In $42 \%$ cases, MRI provided confirmation of the suspected lesions. 


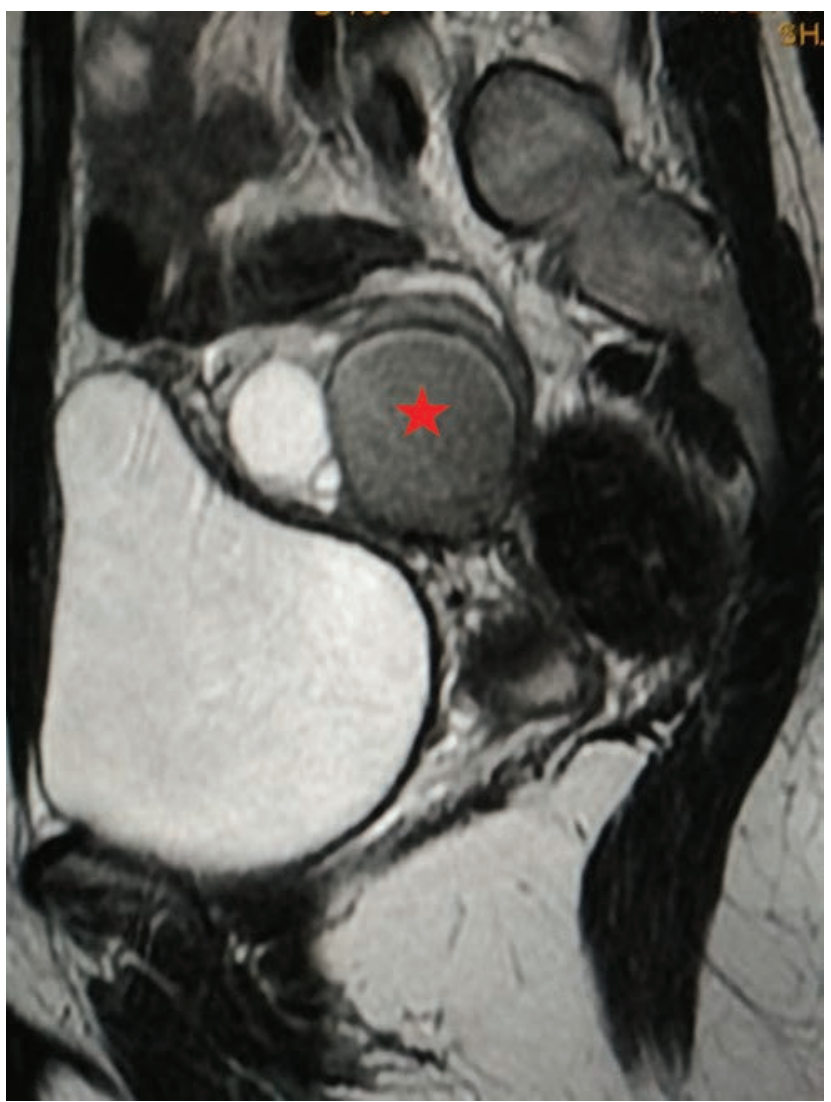

Fig. 12 Sagittal T2-W MRI demonstrating a well-defined cyst (star) with $\mathrm{T} 2$ shading consistent with endometrioma. No evidence of mural nodule/abnormal enhancement. MRI, magnetic resonance imaging.

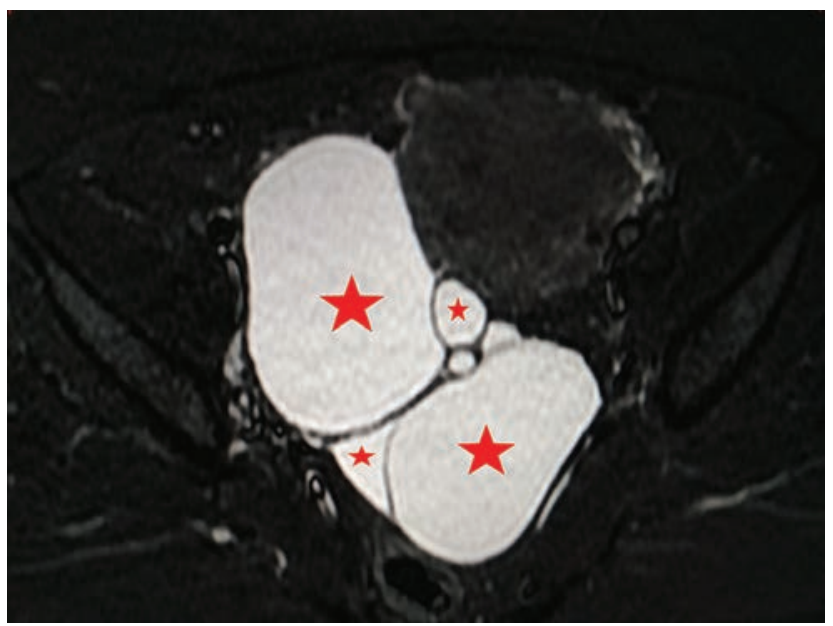

Fig. 13 Axial STIR MRI demonstrating hyperintense cyst of right ovarian origin with multiloculated appearance (stars). No evidence of solid component/hemorrhage. MRI, magnetic resonance imaging.

\section{Conclusion}

In conclusion, pelvic mass lesions in females are more common above 45 years. Multiparity associated with high incidence of pelvic masses. Postmenopausal bleeding has a strong association with the carcinoma cervix. Adnexal masses are usually cystic, while the uterine masses are solid in texture. As the complexity and size of the cystic adnexal masses increases there are increased chances of malignancy. Fat containing masses, such as dermoids, are correctly diagnosed by MRI. Hemorrhagic cyst or intralesional hemorrhage is confidently detected on MRI. MRI is better and accurate in classifying the adnexal masses of female pelvis into benign and malignant. Pretreatment staging and assessment of malignancy, invasion of surrounding structures, encasement, invasion of vessels or assessment of lymphadenopathy, peritoneal implant, ascites, and distant metastases are better appreciated by MRI.

\section{Conflict of Interest}

None declared.

\section{References}

1 Szklaruk J, Tamm EP, Choi H, Varavithya V. MR imaging of common and uncommon large pelvic masses. Radiographics 2003;23(2):403-424

2 Smorgick N, Maymon R. Assessment of adnexal masses using ultrasound: a practical review. Int J Womens Health 2014;6:857-863

3 Kurman RJ, ed. Blaustein's pathology of the female genital tract. vol. 1, 4th ed. New York, NY: Springer-Verlag; 1994

4 Sala E, Wakely S, Senior E, Lomas D. MRI of malignant neoplasms of the uterine corpus and cervix. AJR Am J Roentgenol 2007;188(6):1577-1587

5 Meire HB, Farrant P, Guha T. Distinction of benign from malignant ovarian cysts by ultrasound. Br J Obstet Gynaecol 1978;85(12):893-899

6 Jain KA, Jeffrey RB Jr. Evaluation of pelvic masses with magnetic resonance imaging and ultrasonography. J Ultrasound Med 1994;13(11):845-853

7 Troiano R, Lazzarini K, Scoutt L, McCarthy SM. Ovarian fibromas: MR appearance. Radiology 1997;204:795-798 